\title{
ОЧИСТКА ПОВЕРХНОСТИ АЛЮМИНИЕВОЙ МЕТАЛЛИЗАЦИИ ПРИ ПОМОЩИ ПЛАЗМЕННОЙ ОБРАБОТКИ
}

\author{
() 2017 Е. В. Гончаренко, Т. Г. Меньшикова, М. В. Гречкина, Е. Н. Бормонтов \\ Воронежский государственный университет, Университетская пл., 1, 394018 Воронеж, Россия \\ e-mail: zews-00@mail.ru
}

Поступила в редакцию 21.12.2016 г.

\begin{abstract}
Аннотация. Разработана технология плазменного травления алюминиевой металлизации, приводящая к улучшению качества сварного шва и к 100\% присоединению внутреннего вывода к контактным площадкам кристалла программируемой логической интегральной схемы (ПЛИС). Представлены показатели средней шероховатости, наибольшей высоты неровностей профиля, среднеквадратичной шероховатости до и после обработки в плазме. Проведена оценка влияния плазменного травления алюминиевой металлизации на величину усилия, необходимого для отрыва внутреннего вывода, приваренного к контактной площадке ПЛИС.
\end{abstract}

Ключевые слова: плазменная обработка, алюминиевая металлизация, контактная площадка, ультразвуковая сварка, программируемая логическая интегральная схема (ПЛИС).

\section{ВВЕДЕНИЕ}

Надежность полупроводниковых изделий тесно связана с технологией производства и, в особенности, с операциями получения контактных соединений. Одной из наиболее распространенных причин отказов, связанных с процессом производства, является нарушение микросоединений кристалла с корпусом и внутренних выводов с контактными площадками кристалла и корпуса [1]. По данным отечественной и зарубежной литературы от 35 до $60 \%$ всех отказов в радиоэлектронной аппаратуре приходится на долю микросоединений.

Соединения в полупроводниковых приборах и ИС должны удовлетворять следующим требованиям: прочность соединения должна быть близка к прочности соединяемых элементов микросхем; соединение должно иметь минимальное омическое сопротивление; основные параметры процесса соединения (температура нагрева, удельное давление и длительность выдержки) должны быть минимально возможными с тем, чтобы не повреждались элементы схемы; выполнять соединение материалов разнообразных сочетаний и типоразмеров; после соединения не должно оставаться материалов, вызывающих коррозию; качество соединений должно контролироваться простыми и надежными методами [2].

Цель работы заключается в подборе оптимального режима плазменного травления для очистки поверхности алюминия от загрязнений.

\section{ОБЪЕКТЫ И МЕТОДЫ ИССЛЕДОВАНИЯ}

В данной работе объектом исследования являлась алюминиевая металлизация контактных площадок кристалла ПЛИС. Толщина алюминия в среднем составляла 1 мкм. Размеры контактной площадки 100х100 мкм. Количество контактных площадок на ПЛИС составляет 256 шт.

Очистка поверхности алюминия проводилась при помощи системы плазменной обработки АР1000 в среде аргона.

Контроль влияния плазмы на алюминиевую металлизацию, находящуюся на контактных площадках ПЛИС, проводился на установке разварки тонкой проволокой ВЈ-820 путем приваривания алюминиевой проволоки к контактным площадкам.

Морфология поверхности исследовалась методом сканирующей силовой микроскопии в полуконтактном режиме на сканирующем зондовом микроскопе (C3M) Solver P47 Pro. При сканировании использовались композитные поликремниевые кантилеверы HA_NC Etalon с радиусом закругления острия зонда 10 нм и резонансными частотами 145 и 232 кГц. Неоднородные свойства поверхности контролировались методом отображения фазы.

\section{ЭКСПЕРИМЕНТАЛЬНАЯ ЧАСТЬ}

Прочность соединения и его повторяемость напрямую зависит от типа, материала поверхности и проводника, диаметра привариваемого провод- 
ника, выбранных режимов сварки и чистоты поверхности [3].

Для того чтобы избежать отслоений, повысить качество, надежность и повторяемость сварных соединений в данной работе использовалась плазменная обработка поверхностей перед началом создания проволочных выводов в операции сборки ПЛИС. Плазменная обработка сводится к очистке и модификации поверхности, что позволяет вносить локальные изменения в свойства обрабатываемой поверхности (гидрофильность, адгезионные характеристики, химический состав и т. д.) при сохранении неизменными объемных свойств. Основными плазмообразующими средами в данном случае служат $\mathrm{Ar}, \mathrm{O}_{2}, \mathrm{NO}, \mathrm{NH}_{3}$, их смеси и воздух.

Очистка поверхности алюминия проводилась при помощи системы плазменной обработки
АР1000 в среде аргона, т.к. использование в плазме кислорода ускоряет процесс окисления поверхности алюминия. Эффективность очистки аргоновой плазмой в первую очередь связана с большим размером атомов аргона. Масса атомов аргона почти в два раза превышает массу атомов кислорода. По этой причине атомы аргона могут ударяться о поверхность образца с большей силой (энергией). Поэтому физический процесс очистки требует более высокой мощности и низкого давления (150-300 мТорр). Суть метода состоит в придании максимального ускорения атомам и ионам аргона перед тем, как они столкнутся с поверхностью образца. Высокая мощность необходима по той причине, что при большей энергии плазмы будет больше средняя скорость атомов и ионов в плазме. Низкое давление необходимо для
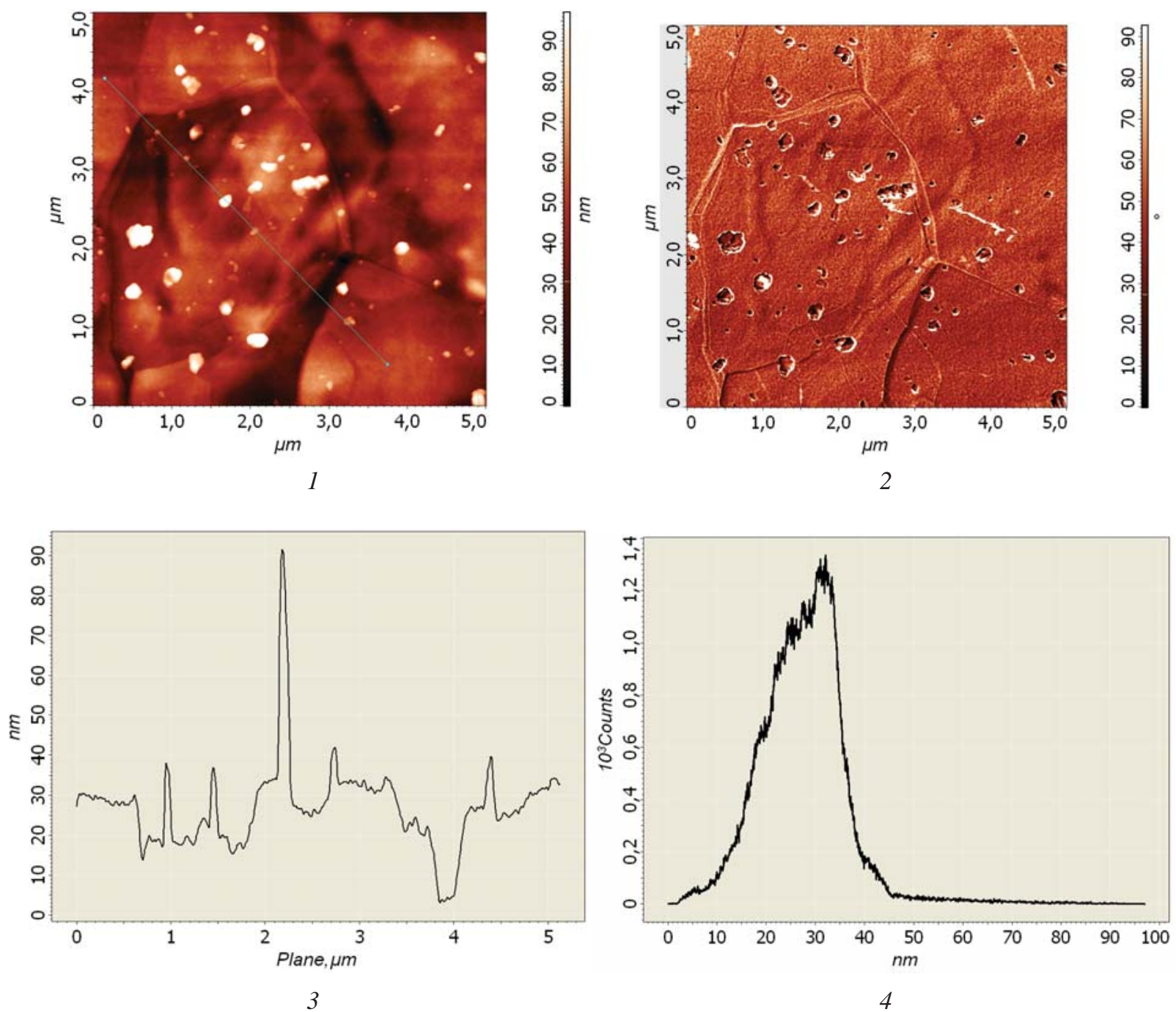

Рис. 1. АСМ-изображение поверхности пленки алюминия до обработки в плазме: 1) топография поверхности;

2) фаза; 3) сечение рельефа; 4) гистограмма

[Fig. 1. AFM-image of a surface of a film of aluminum before processing in plasma: 1) surface topography; 2) phase; 3) contour interval; 4) histogram] 
увеличения среднего расстояния, которое каждый атом проходит до столкновения с другим атоMOM.

Образцы ПЛИС № 1, № 2, № 3, № 4 были подвергнуты обработке в плазме в 4-х разных режимах. Режим № 1 описывается в работе Comparison OfArgon And Oxygen Plasma Treatments: 350 W, вре- мя от 45 до 180 секунд, поток - 40 мл/м [4]. Режим № 2 описан в работе Process Windows for Low-temperature Au Wire Bonding: 100 Ватт при давлении 120 мторр с потоком газа 5 мл/м в течение 5 минут [5]. Режим № 3 описан в работах американской компании Micron: 550 Ватт, время - 30 секунд, давление 160 мторр, поток - 40 мл/м. Режим № 4

Таблица 1. Режимы проведения обработки в плазме

[Table 1. The modes of plasma treatment]

\begin{tabular}{|c|c|c|c|c|}
\hline $\begin{array}{c}\text { Режим № } \\
{[\text { Mode No] }}\end{array}$ & $\begin{array}{c}\text { Мощность, Ватт } \\
{[\text { Power, Watt] }}\end{array}$ & $\begin{array}{c}\text { Время, с } \\
{[\text { Time, } \mathrm{s}]}\end{array}$ & $\begin{array}{c}\text { Давление, мТорр } \\
{[\text { Pressure, mTorr] }}\end{array}$ & $\begin{array}{c}\text { Поток, мл/м } \\
{[\text { Stream, } \mathrm{ml} / \mathrm{m}]}\end{array}$ \\
\hline 1 & 350 & 100 & 150 & 40 \\
\hline 2 & 100 & 300 & 120 & 5 \\
\hline 3 & 550 & 30 & 160 & 40 \\
\hline 4 & 510 & 800 & 150 & 40 \\
\hline
\end{tabular}

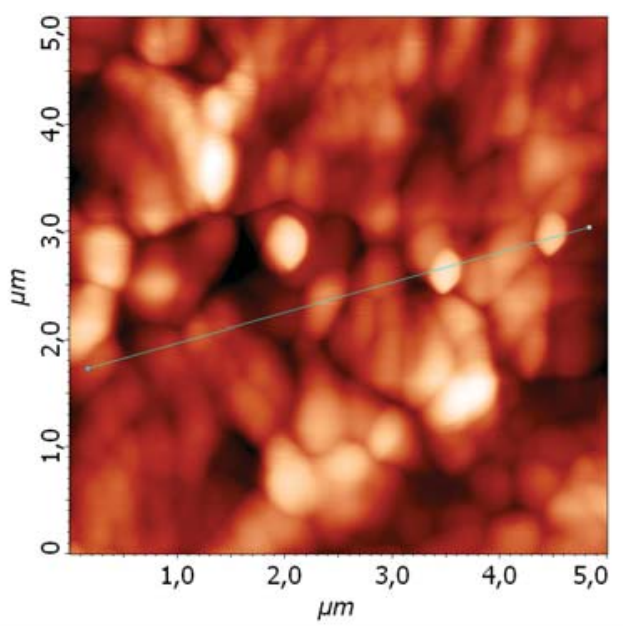

1

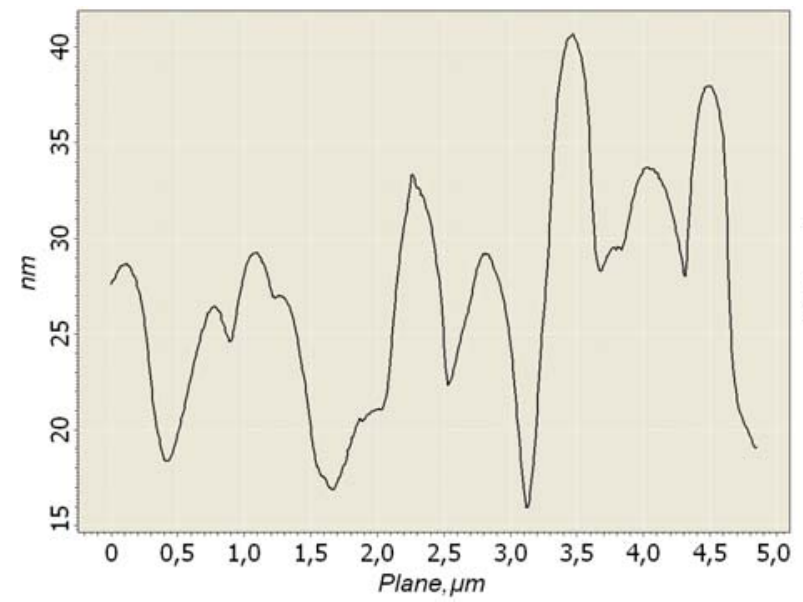

3
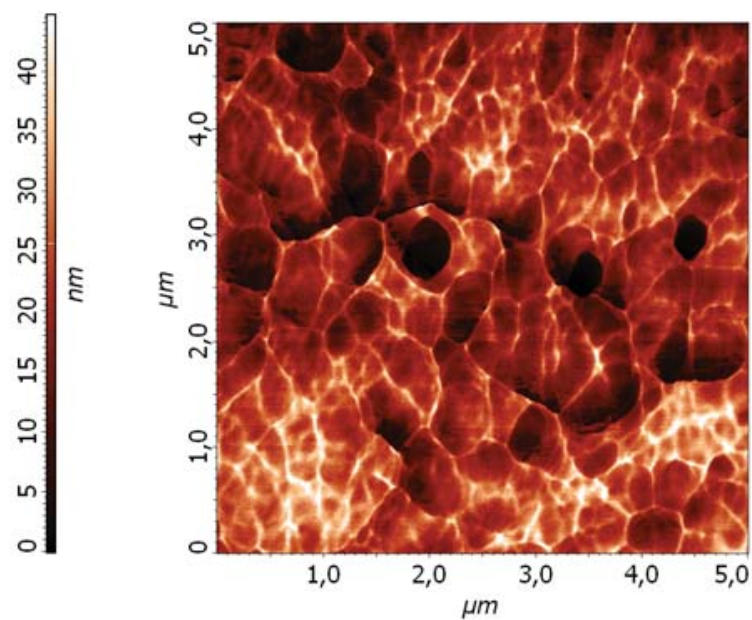

2

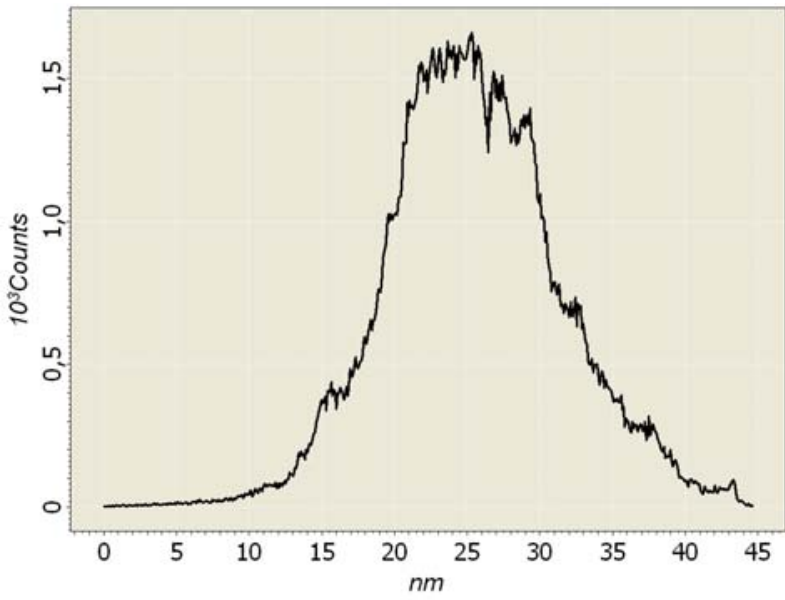

4

Рис. 2. АСМ-изображение поверхности пленки алюминия после обработки в плазме: 1) топография поверхности; 2) фаза; 3) сечение рельефа; 4) гистограмма

[Fig. 2. ASM-image of a surface of a film of aluminum after processing in plasma: 1) surface topography; 2) phase; 3) contour interval; 4) histogram] 
подбирался экспериментальным путем, опираясь на теоретические данные.

Элементный состав пленок определялся с помощью системы энергодисперсионного анализа X-flash (Bruker).

Для выявления изменений после обработки в плазме рассчитывалась шероховатость. Расчет шероховатости до и после обработки в плазме проводился следующим образом. $R_{a}$ - средняя шероховатость определяется как среднее арифметическое отклонение профиля от средней наклонной прямой или плоскости, проведенной методом наименыших квадратов [6]. Этот параметр определяется как площадь отклонения профиля шероховатости относительно средней прямой (плоскости), деленной на общую длину базовой линии, и численно равен интегралу:

$$
R_{a}=\frac{1}{L} \int_{0}^{L}|r(x) d x|
$$

$R_{q}$ - среднеквадратичная шероховатость характеризует среднеквадратичное отклонение профиля поверхности относительно базовой линии и вычисляется как

$$
R_{q}=\sqrt{\frac{1}{L} \int_{0}^{L} r(x) d x} .
$$

$R_{\max }$ - наибольшая высота неровностей профиля или расстояние между наибольшим пиком и наименьшей впадиной на базовой линии (плоскости).
Результат расчета шероховатости до и после обработки в плазме приведен в табл. 3 .

По результатам расчета шероховатости можно сделать вывод, что величина средней шероховатости, наибольшей высоты неровностей профиля, среднеквадратичной шероховатости после обработки в плазме стала меньше.

Дополнительный контроль влияния плазмы на алюминиевую металлизацию, находящуюся на контактных площадках ПЛИС, проводился на установке разварки тонкой проволокой ВЈ-820 путем приваривания алюминиевой проволоки к контактным площадкам.

После обработки в плазме на каждом из 4-х образцов было разварено по 10 сварных соединений. Разварка проводилась алюминиевой проволокой диаметром 35 мкм.

Контроль прочности сварных соединений проводился граммометром часового типа. Результаты приведены в табл. 4. $N_{1}-7.6(г), N_{2}-5$ (г), $N_{3}-8.9$ (г), $N_{4}-12.6$ (г), где $N_{1}, N_{2}, N_{3}, N_{4}$ - средние отрывные усилия сварных соединения при плазменном травлении на режимах № 1, 2, 3, 4 соответственно.

Исходя из полученных данных, следует, что отрывные усилия сварных соединений образца № 4, обработанного на подобранном режиме обработки в плазме, намного выше, чем отрывные усилия первых 3-х образцов.

Таблица. 2. Процентное содержание химических элементов в контактной площадке

[Table. 2. Percentage of chemical elements in the contact platform]

\begin{tabular}{|c|c|c|c|}
\hline \multicolumn{2}{|c|}{$\begin{array}{c}\text { До обработки в плазме } \\
\text { [Before processing in plasma] }\end{array}$} & $\begin{array}{c}\text { После обработки в плазме } \\
\text { [After processing in plasma] }\end{array}$ \\
\hline $\begin{array}{c}\text { Химический элемент } \\
\text { [Chemical element] }\end{array}$ & $\mathrm{Wt.} \%$ & $\begin{array}{c}\text { Химический элемент } \\
\text { [Chemical element] }\end{array}$ & Wt. \% \\
\hline $\mathrm{C}$ & 5.708717 & $\mathrm{C}$ & 5.172423 \\
\hline $\mathrm{O}$ & 5.597612 & $\mathrm{O}$ & 5.056324 \\
\hline $\mathrm{Al}$ & 52.03568 & $\mathrm{Al}$ & 51.73456 \\
\hline $\mathrm{Si}$ & 13.48945 & $\mathrm{Si}$ & 13.47583 \\
\hline $\mathrm{Ar}$ & 0.570771 & $\mathrm{Ar}$ & 1.050891 \\
\hline $\mathrm{Ti}$ & 12.23532 & $\mathrm{Ti}$ & 12.34285 \\
\hline $\mathrm{W}$ & 10.36245 & $\mathrm{~W}$ & 11.16712 \\
\hline
\end{tabular}

Таблица. 3. Расчет шероховатости алюминиевой металлизации до и после обработки в плазме

[Table 3. Calculation of aluminum metallization roughness before plasma treatment]

\begin{tabular}{|c|c|c|c|}
\hline & $R_{a}, \mathrm{~nm}$ & $R_{\max }, \mathrm{nm}$ & $R_{q}, \mathrm{~nm}$ \\
\hline $\begin{array}{c}\text { До обработки в плазме } \\
\text { [Before processing in plasma }]\end{array}$ & 7.45 & 194 & 10.8 \\
\hline $\begin{array}{c}\text { После обработки в плазме } \\
\text { [After processing in plasma] }\end{array}$ & 6.05 & 59.2 & 7.84 \\
\hline
\end{tabular}


Таблица 4. Отрывные усилия сварных соединений

[Table 4. Detachable efforts of wire bonds]

\begin{tabular}{|c|c|c|c|c|c|c|c|c|c|c|}
\hline & \multicolumn{10}{|c|}{$\begin{array}{l}\text { Отрывное усилие сварного соединения, Г } \\
\text { [Detachable effort of welded connection, g] }\end{array}$} \\
\hline $\begin{array}{l}\text { Образец № } \\
\text { [No. sample] }\end{array}$ & 1 & 2 & 3 & 4 & 5 & 6 & 7 & 8 & 9 & 10 \\
\hline 1 & 9 & 7 & 7 & 9 & 8 & 8 & 6 & 7 & 7 & 8 \\
\hline 2 & 5 & 6 & 5 & 4 & 6 & 5 & 6 & 4 & 5 & 4 \\
\hline 3 & 10 & 9 & 10 & 8 & 9 & 8 & 10 & 9 & 8 & 8 \\
\hline 4 & 13 & 14 & 12 & 12 & 13 & 14 & 14 & 13 & 12 & 12 \\
\hline
\end{tabular}

Таким образом, был подобран оптимальный режим обработки в плазме, при котором улучшилось качество сварного соединения между контактной площадкой и внутренним соединением ПЛИС.

\title{
ВЫВОДЫ
}

1. Разработана технология обработки поверхности алюминиевой металлизации путем плазменного травления, приводящей к $100 \%$ присоединению внутреннего вывода к контактной площадке ПЛИС.

2. Представленная технология обработки поверхности алюминиевой металлизации позволяет увеличить усилие, необходимое для отрыва внутреннего вывода, приваренного к контактной площадке ПЛИС, на $20 \%$.

Результаты исследований получены на оборудовании Центра коллективного пользования научным оборудованием ВГУ.

\section{СПИСОК ЛИТЕРАТУРЫ}

1. Емельянов В. А. Корпусирование интегральных схем. Минск, Полифакт, 1998, 360 с.

2. Ланин В., Петухов И. // Компоненты и технологии, 2009, № 8, с. 124-128.

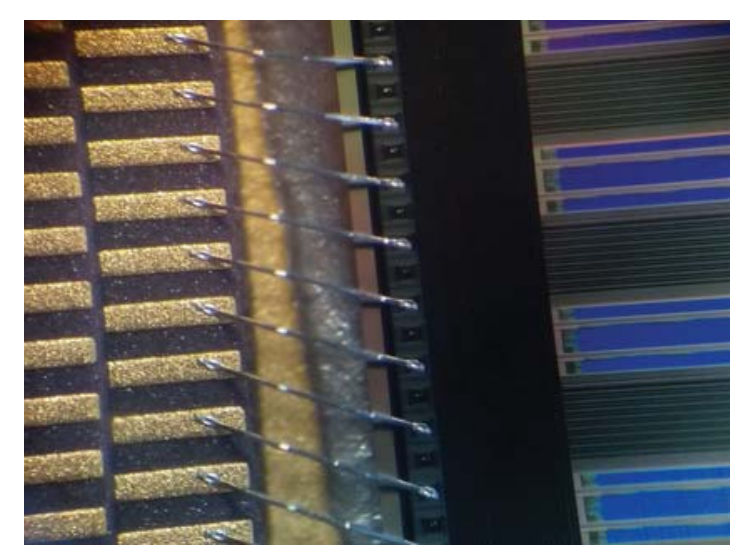

Рис. 3. Изображение внутренних выводов с качественным сварным соединением

[Fig. 3. Image of internal pins with high-quality bonds]

3. Гасанов И. С. // Плазменная и пучковая технология, 2007, с. 125.

4. Hui Yuen Peng, Mutharasu Devarajan, Teik Toon Lee // International Journal of Scientific \& Engineering Research, 2014, vol. 5, iss. 12, pp. 908-912.

5. Yu Hin Chan, Jang-Kyo Kim, Deming Liu // J. of Electronic Materials, 2004, vol, 33, iss, 2, pp. 146-155.

6. Арутюнов П. А., Толстихина А. Л., Демидов В. И. // Заводская лаборатория, 1999, т. 65, № 9, с. 31-41.

\section{ALUMINUM METALLIZATION SURFACE CLEANING WITH PLASMA TREATMENT}

\author{
(C) 2017 E. V. Goncharenko, E. N. Bormontov, T. G. Menshikova, M. V. Grechkina \\ Voronezh State University, Universitetskaya sq., 1, 394018 Voronezh, Russia \\ e-mail: zews-00@mail.ru
}

Received 21.12.2016

Abstract. The purpose of work is to select the optimum plasma etching mode for cleaning the aluminum surface from pollution. 
METHODS AND METHODOLOGY. In this paper, FPGA chip`s pads aluminum metallization was the object of the study. The averaged thickness of aluminum was 1 micron. The contact pads sizes $100 \times 100$ microns. FPGA got 256 contact pads.

AP-1000 Plasma Treatment System accommodated with the argon gas used for aluminum surface cleaning.

Plasma influence control on the aluminum metallization, located on the FPGA pads, was carried out with the Bondjet BJ820 bonding the aluminum wire to the pads.

The surface morphology was investigated by scanning force microscopy in semi-contact mode by a scanning probe microscope (SPM) Solver P47 Pro.

RESULTS. The optimum mode of plasma treatment which gives quality bonds between the contact pads and the FPGA internal connection was obtained.

CONCLUSIONS:

1. The new technology of the aluminum metallization surface treatment using plasma etching with the $100 \%$ internal pin to the FPGA contact pads bonding was developed.

2. The presented technology of an aluminum metallization surface treatment allows to improve quality of the separation effort to the internal pin welded on the FPGA contact platform up to $20 \%$.

Keywords: plasma treatment; aluminum metallization; pad; ultrasonic welding; FPGA.

\section{ACKNOWLEDGEMENTS}

The research results were obtained with equipment of Voronezh State University Centre for Collective Use of Scientific Equipment.

\section{REFERENCES}

1. Emelyanov V. A. Encapsulation of Integrated Circuits. Minsk, Polifakt Publ., 1998, 360 p. (in Russian)

2. Lanin V. Petukhov I. Components and Technologies, 2009, no. 8, pp. 124-128. (in Russian)

3. Gasanov I. S. Plasma and Beam Technology, 2007, p. 125. (in Russian)
4. Hui Yuen Peng, Mutharasu Devarajan, Teik Toon Lee. International Journal of Scientific \& Engineering Research, 2014, vol. 5, iss. 12, pp. 908-912. Available at: (http://www. ijser.org/researchpaper/Comparison-of-Argon-and-OxygenPlasma-Treatments-on-LED-Chip.pdf

5. Yu Hin Chan, Jang-Kyo Kim, Deming Liu. J. of Electronic Materials, 2004, vol. 33, iss. 2, pp. 146-155. DOI: $10.1007 / \mathrm{s} 11664-004-0285-5$

6. Arutyunov P.A., Tolstikhina A.L., Demidov V.I. Zavodskaya Laboratoriya. Diagnostika Materialov, 1999, vol. 65, no. 9, pp. 31-41. (in Russian)
Гончаренко Евгений Владимирович - аспирант 1 года обучения физического факультета, Воронежский государственный университет; e-mail: zews-00@mail.ru

Меньшикова Татьяна Геннадьевна - к. ф.-м. н., доцент кафедры полупроводников и микроэлектроники, Воронежский государственный университет; e-mail: menshikova.vrn@mail.ru

Гречкина Маргарита Владимировна - инженер ЦКП НО ВГУ, Воронежский государственный университет; e-mail: grechkina_m@mail.ru

Бормонтов Евгений Николаевич - д. ф.-м. н., профессор, заведующий кафедрой физики полупроводников и микроэлектроники, Воронежский государственный университет; тел.: +7 (473) 2208481, e-mail: me144@ phys.vsu.ru
Goncharenko Evgeny V. - 1 year post-graduate of Physical Faculty, Voronezh State University; e-mail: zews00@ mail.ru

Menshikova Tatiana G. - Cand. Sci. (Phys.-Math.), Associate Professor, Department of Semiconductor and Microelectronics, Voronezh State University; e-mail: menshikova.vrn@mail.ru

Grechkina Margarita V. - Engineer of the Center for Collective Use of Scientifie Equipment, Voronezh State University; e-mail: grechkina_m@mail.ru

Bormontov Eugene N. - Dr. Sci. (Phys.-Math.), Head of Department of Physics of Semiconductors and Microelectronics, Voronezh State University; tel.: +7 (473) 2208481, e-mail: me144@phys.vsu.ru 